\title{
Gas Hydrates of Coal Layers as a Methane Source in the Atmosphere and Mine Working
}

\author{
Valery Dyrdin ${ }^{1}$, Sofya Shepeleva $^{{ }^{*}}$, and Tatiana $\mathrm{Kim}^{1}$ \\ ${ }^{1}$ T.F. Gorbachev Kuzbass State Technical University, 650000, 28 Vesennyaya St., Kemerovo, Russia
}

\begin{abstract}
Living conditions of gas hydrates of a methane in a coal matrix as one of possible forms of finding of molecules of a methane in coal layers are considered. However, gas hydrates are formed not in all mineral coals even under the thermobaric conditions corresponding to their equilibrium state as the minimum humidity and the corresponding pore width are necessary for each brand of coal for formation of gas hydrate. It is shown that it depends on electric electrical dipole moment of a macromolecule of coal. Coals of brands K, D, Zh were considered. The electric field created by the surface of coal does not allow molecules of water to carry out threedimensional driving, and they keep on an internal surface of a time. By means of theoretical model operation a dipole - dipole interaction of molecules of water with the steam surface of coal values of energy of fiber interaction for various functional groups located in coal "fringe" which size for the first and second layers does not allow molecules of water to participate in formation of gas hydrates are received. For coals of brands K, $\mathrm{Zh}, \mathrm{D}$, considering distribution of a time on radiuses, the percent of moisture, which cannot share in education solid coal of gas solutions, is calculated.
\end{abstract}

\section{Introduction}

By underground mining of gas-bearing coalfields in some cases there are sudden emissions of coal and gas - the catastrophic natural phenomena accompanied with emission of hundreds of tons of the crushed coal and tens of thousands of cubic meters of natural gas. Not only destruction of excavations, cars and mechanisms, but also a deadly traumatizing of miners follow them. In spite of the fact that coal layer from a particular depth can be carried to threatened or dangerous on sudden emissions of coal and gas, emissions happen irregularly and not on all territory of the mine field, and in particular points. Apparently, it is bound to padding factors, which still were not considered when developing the theory of sudden emissions of coal and gas and methods of their prevention.

For practice of mining and environment protection against harmful emissions it is important to know not only total of the methane which is contained in coal, but also its phase state, that is a ratio between amounts of methane in the form of the free gas and a methane in other phase states. The role of various forms of existence of a methane in coal substance in formation of gas of dynamic processes is up to the end not clear, but from practice, it is known that the volume of the methane, which is allocated at the same time, exceeds natural gas

* Corresponding author: shepelevasa@kuzstu.ru 
content. The thermodynamically coordinates assuming existence in coal layers of solid solutions of natural gases as gas hydrates are characteristic of the existing development depths in mines of Kuzbass. The factors promoting existence of gas in the form of gas hydrates in natural system are existence in reservoir gases of mixes of hydrocarbons and impurity of other gases, natural moisture, particular thermo baric parameters and also high mechanical tensions which at a depth of $500 \mathrm{~m}$ are about $12,5 \mathrm{MPa}$ (and in zones of tectonic violations of tension can exceed this size [1]). It is possible to consider the following forms of finding of a methane in coal layers [2]:

- the free state in cracks and a time of coal. In this case the coal-gas system submits to laws of a condition of real gas;

- condition of the surface (physical) adsorption; this case the system submits to the law of Lengmyur, i.e. the amount of stripped gas is proportional to its pressure;

- the macromolecule of coal forms a chemical bond with molecules of gases, that is between an adsorbent and an adsorbate electron transfer takes place. Such type of communication is called chemical adsorption, or a chemosorption;

- crystallohydrate or just gas hydrate of a methane; fragile crystal connections which are usually unstable also exist in a coal matrix under particular thermo dynamic conditions[3].

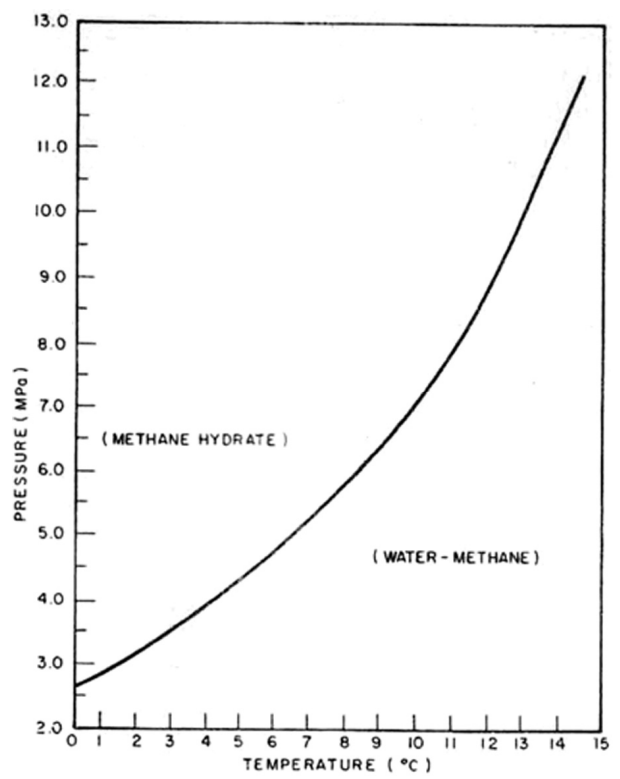

Fig. 1. Thermobaric zones of possible decomposition of hydrate of a methane on phases gas/ice and gas / the overcooled water at a depressurizing to various values [8].

Therefore, the gas balance of sudden emissions has to be presented by the sum of the free gas concluded in a coal pore space; the gas occluded on a surface of cracks and macropores, and gas, which is emitted at dissociation of gas hydrates.

\section{Materials and methods}

Earlier A.G. Zenin, 1973; I.L. Oettinger, 1974; Yu.F. Makogon, 1973 suggested that a methane in mineral coal under particular thermo dynamic conditions can be in a type of the gas hydrates representing the solid connections formed by a crystal lattice of molecules of water which cavities are busy with methane molecules. On average, six molecules of water are the share of one molecule of a methane in gas hydrate. The water necessary for formation 
of hydrate of a methane is in a coal time and is connected by electrostatic forces with the surface of coal. Gas which is in the free state and water under particular thermobaric conditions practically is always formed by gas hydrates. But in a coal matrix of a molecule of gas and water are polarized under the influence of electrical dipole moment [4-6]. In the course of formation of gas hydrate water molecules which energy exceeds bond energy with the surface of coal can participate. Therefore, for the solution of a question of formation of gas hydrates in a coal matrix it is necessary to compare interaction energy of molecules of water with a surface of a coal matrix and energy of thermal motion.

Depending on thermodynamical parameters system moisture - methane can be in a state gas - hydrate (fig. 1) or gas - moisture, and states are divided by an equilibrium curve [7]. At a temperature about $10{ }^{\circ} \mathrm{C}$ transition of a methane from a hydrated state in gaseous is possible at sharp pressure drop from values above of $8 \mathrm{MPa}$ (over a curve of phase change) to values of smaller $6 \mathrm{MPa}$ (below a curve).

It is experimentally established that gas hydrates are formed not in all mineral coals, even under the corresponding thermobaric conditions. For coals of different extent of metamorphism there is a minimum humidity at which gas hydrates are formed [9].

The crystal lattice of coal represents graphite similar grids (fig. 2) consisting of hexangular rings. Electrical dipole moment of coals depends on functionality, contained in "fringe" of a macromolecule of coal, their concentration and the location on a surface. A task of this article is establishment of a possibility of formation of gas hydrates of a methane in coals of a different stage of metamorphism depending on their electrical dipole moment and pore size.

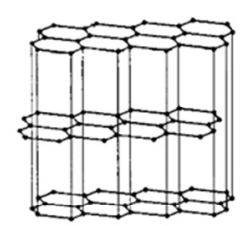

$a$

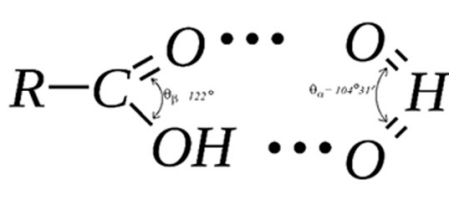

$b$

Fig. 2. Model of interaction of the functional groups on the surface of coal with a water molecule: $a-$ a coal crystal structure, a general view; $b$ - an illustration of designations at record of potential of Shtokmayer on the example of the functional group of $\mathrm{R}-\mathrm{COOH}$ and a molecule of water.

Let's consider model of interaction of molecules of water from one of the functional groups which is contained on a carbon surface. The model is limited to consideration of pair interaction of the molecule $\mathrm{H} 2 \mathrm{O}$ and one of the functional groups, for example, by $\mathrm{COOH}$ (fig. 2). Molecules of water are caught by the surface of coal and lose an opportunity to carry out a three-dimensional Brownian motion. The mobility of the adsorbed molecules is limited to their twodimensional movement on a surface; molecules keep on an internal surface of a time, but do not get into a field of force of atoms and molecules of coal $[10,11]$. In most cases at the quantitative description use so-called potentials of an intermolecular interaction. One of widespread potentials of pair interaction of the molecules having constant electrical dipole moment is potential of Shtokmayer [12]:

$$
\begin{gathered}
U\left(r, \theta_{\alpha}, \theta_{\beta}, \varphi\right)=4 \varepsilon\left(\left(\frac{\sigma}{r}\right)^{12}-\left(\frac{\sigma}{r}\right)^{6}\right)-\frac{\mu_{\alpha} \mu_{\beta}}{r^{3}} g, \\
g=2 \cos \theta_{\alpha} \cos \theta_{\beta}-\sin \theta_{\alpha} \sin \theta_{\beta} \cos \varphi,
\end{gathered}
$$


where $\varepsilon$ - characteristic energy, J; $\sigma$ - characteristic length, nanometer; $r$ - center distance of the interacting molecules, nanometer; $\mu_{\alpha}, \mu_{\beta}$ - electrical dipole moments of molecules, $\mathrm{Kl} \cdot \mathrm{m} ; \theta_{\alpha}, \theta_{\beta}$ - polar angles of molecules, degree; $\varphi$ - difference of lateral angles of molecules, degree.

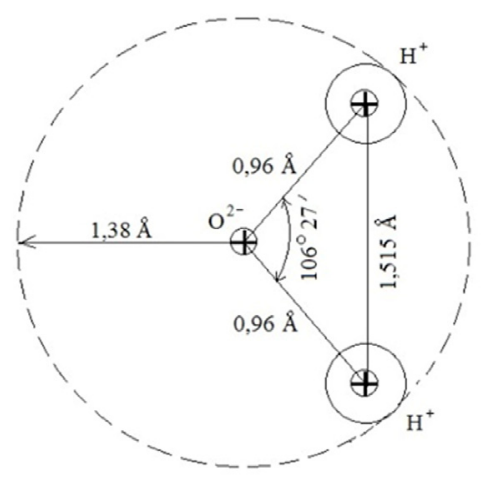

Fig. 3. Water molecule mode.

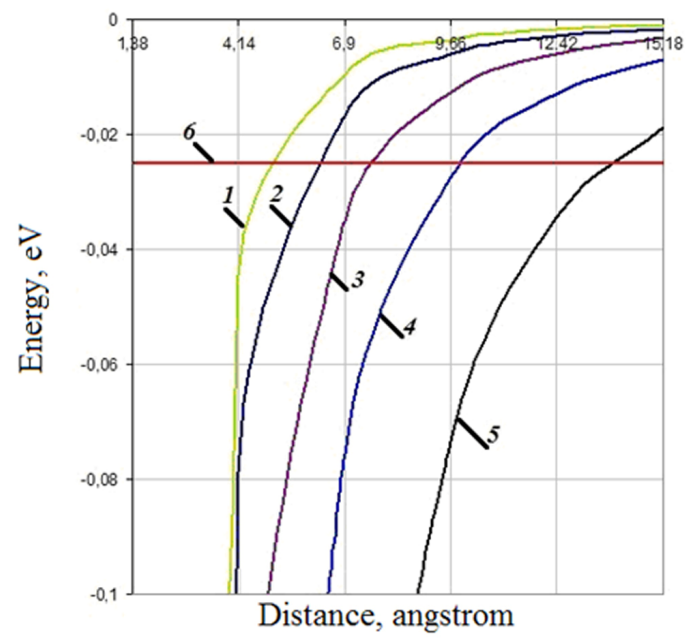

Fig. 4. Dependence of interaction energy of the functional groups on the surface of coal with water molecules from distance: 1 - with the $\mathrm{C}$-O group; 2 - with the $\mathrm{CH}_{3}$ group; 3 - with the $\mathrm{C}-\mathrm{H}$ group; 4 with the COON group; 5 - with the $\mathrm{C}=\mathrm{O}$ group; 6 - thermal energy at $\mathrm{T}=286 \mathrm{~K}$

For the qualitative description of interaction of a molecule of water with the surface of coal we use model of Bernal and Fowler (fig. 3). According to the considered model, the center of the negative charge is located in the center of the sphere which radius is equated to the efficient radius of the molecule $\mathrm{H}_{2} \mathrm{O}$ and makes size about 0,138 nanometers. As a first approximation, the water molecules which are immediately near the surface of coal are polarized along the field created by this surface. And at some distance from a surface polarization of molecules of water will be defined by a density fluctuation of distribution of volume negative charge or influence of the next polarized molecules.

\section{Results and discussion}


With use of potential of Shtokmayer was calculated energy of fiber interaction for each functional group with water molecules. In fig. 4 it is visible that for the first coat of molecules of water located apart 4,14 angstrom, energy of thermal agitation modulo is less than energy of dipole interaction of molecules of water with the functional groups on the surface of coal. Therefore, it is impossible to tear off the water molecules located in the first coat of rather coal surface.For different functional groups dipole-dipole energy will be various. The method ${ }^{13} \mathrm{C}$ of a nuclear magnetic resonance spectroscopy obtained the data reflecting changes in structure of coals of various extent of metamorphism $[13,14]$. Several functional groups are allocated, we will consider only those which have electrical dipole moment: $\mathrm{CH}_{3}$ (electrical dipole moment 0,4 D); $\mathrm{OCH}_{3}$ (1,3 D); C-H (0,4 D); C-O (2,5 D); $\mathrm{COOH}(1,63 \mathrm{D})$; $\mathrm{C}=\mathrm{O}(2,7$ $\mathrm{D})$. Using data [13] was calculated that for brand $\mathrm{D}$ coals electrical dipole moment can change within $0,04-0,17$ of D; for coals of the Zh 0,03 - 0,28 D brand; for coals of the K 0,02 - 0,28 D brand.

Comparing the radius of molecules of water and cross sectional dimension of a time, it was received that in micropores (cross sectional dimension up to 0,6 nanometers) there can be only two molecules of water that corresponds to one layer; in the transitional time (cross sectional dimension of 1 - 10 nanometer) of 1 - 18 layers of molecules of water; in macropores (cross sectional dimension of 10 - 100 nanometers) of 1 - 180 layers of molecules of water. Considering distribution of a time, for coals of different brands the percent of moisture which cannot share in formation of gas hydrates is calculated.

\section{Conclusion}

1. It is established that for coals of the considered brands the moisture which is in micropores cannot take part in these processes. For brand D coals from transitional and macropores of a time it is not possible to use $33 \%$ of moisture. For coals of brand $\mathrm{Zh}$ and $\mathrm{K}$ up to $83 \%$.

2. It is shown that at the uniform distribution of water on a time at natural humidity of $2 \%$ in coals with porosity of $10 \%$ education up to $132 \mathrm{~g}$ of hydrate of a methane on $1 \mathrm{t}$ of coal is possible. In coal layers the congestion of gas hydrates represents not a monolithic piece, but distribution in structure of coal layer in the form of the particles comparable to pore sizes. Decomposition of hydrate in a porous medium or in selfcontained volume leads to the substantial increase of gas pressure. At the same time it can be allocated up to $160-$ 180 volumes of pure gas that asks decrease in a share of a methane as greenhouse gas in gas balance of sudden emissions.

\section{References}

1. D. R. Hanson, Mining Engeneering, 37:1, 54 (1985)

2. A.D. Alexeev, T.A. Vasylenko, SOLID STATE COMMUN, 130:10, 669 (2004)

3. S. A. Shepeleva, V. V. Dyrdin, Journal of Mining Science, 47:5, 660 (2011)

4. Dimo Kashchieva, Abbas Firoozabadib, Journal of Crystal Growth, 243, 476 (2002)

5. P. Claudio, Jr. Ribeiro, L.C. Paulo, Chemical Engineering Science, 63, 2007 (2008)

6. Matthew Clarke, P. Ray Bishnoi, The Canadian Journal of Chemical Engineering, 79, $143(2001)$

7. V. G. Smirnov, V. V. Dyrdin, Z. R. Ismagilov, Fuel, 166, 188 (2016)

8. Christophe Dicharry, Pascal Gayet, Gerard Marion, Alain Graciaa and Anatoliy Nesterov, J. Phys. Chem., 109, 17205 (2005)

9. T. Kim, V. Dyrdin, V. Smirnov, E3S Web of Conferences, 15, 01022 (2017) 
10. I. L. Ettinger, Russian Chemical Reviews, 34:7, 496 (1965)

11. E. R. Khabibulina, Z. R. Ismagilov, N. V. Zhuravleva, Workshop on Black Carbon, 20 (2016)

12. N. I. Fedorova, T. S. Manina, Z. R. Ismagilov, Solid Fuel Chemistry, 48:4, 245 (2014) 